\title{
La credibilidad de las redes sociales en el ámbito periodístico'
}

\author{
The credibility of social media \\ in journalism
}

Eva Herrero CURIEL²

\section{Resumen}

Las redes sociales forman parte de las rutinas periodísticas de cualquier medio de comunicación. Los responsables de las organizaciones informativas desarrollan acciones y estrategias que contemplan los usos de las redes sociales en el entorno profesional. Sin embargo, y a pesar de su penetración, el nivel de confianza que los periodistas depositan en estas plataformas es bajo si se compara con el que tienen respecto a los medios tradicionales. El presente artículo es una investigación que tiene como objetivo conocer el uso que los periodistas españoles hacen de las redes sociales en su trabajo diario. Partiendo de la hipótesis de que las redes sociales siguen sin generar suficiente confianza entre los periodistas, a pesar de queéstos recurren a ellas para buscar información y contactar con fuentes, se ha administrado una encuesta online a 410 periodistas españoles (prensa escrita, televisión, radio, web, gabinetes y freelances). Tras constatar que las redes sociales son utilizadas en gran medida por todos ellos, se puede concluir que la confianza que tienen hacia ellas es escasa y siguen fiándose más de los medios tradicionales.

Palabras clave: Fiabilidad. Medios de comunicación. Periodismo. Redes sociales.

\begin{abstract}
Social media is part of the journalistic routines in all mass media. News company-managers develop actions and strategies to include the uses of social media in the professional field. Nevertheless, in spite of their professional penetration, the level of trust that journalists have in these platforms is low compared to traditional mass media. The main objective of this paper is to find out how Spanish journalists are using social media. The hypothesis is that social media doesn't hold enough trust amongst journalists, despite the fact that they use social media to look for information and to contact sources. An online survey, conducted among more than 400 Spanish journalists (newspaper, television, radio, internet and freelancers), showed that social media is used by most Spanish journalists but their trust in these new social channels is lower than in traditional mass media.
\end{abstract}

Keywords: Trust. Mass media. Journalism. Social media.

\section{Introducción}

La presente investigación se desarrolla en el área de los"emisores institucionales"(Wolf, 1987), entendiendo por tales a periodistas o profesionales de los medios que elaboran y difunden mensajes a través de diferentes canales informativos. En torno a esta línea de investigación, dos han sido los temas tradicionalmente presentes en la

\footnotetext{
1 Artículo extraído de la tesis doctoral de E.H. CURIEL, intitulada "Periodistas y redes sociales en España". Universidad Carlos III de Madrid, España, 2013.

2 Universidad Carlos III de Madrid, Facultad de Humanidades, Comunicación y Documentación, Departamento de Periodismo y Comunicación Audiovisual. Calle Madrid, 133, 28903, Getafe, Madrid, España. E-mail:<eva.herrero@uc3m.es>.

Recebido el 6/3/2014, re-presentado el 13/8/2014 y aceptado para su publicación el 12/9/2014
} 
agenda investigadora de la comunicación: por un lado, los emisores desde el punto de vista de sus características sociológicas aquí se han estudiado los factores externos que influyen en los procesos productivos de los comunicadores (cultura, socialización, normas de profesión, etc.); por otro lado, desde una perspectiva comunicacional, se han tratado de estudiar los procesos y dinámicas que guían la construcción de mensajes, como pueden ser los tradicionales estudios sobre gatekeeping y newsmaking (Lewin, 1947; White, 1950; Gieber, 1956; Gans, 1979; Tuchman, 1980; Robinson, 1981; Fishman, 1983; Wolf, 1987; McQuail, 2000). Nuestro interés radica en esa parte más comunicacional, donde las rutinas periodísticas y los usos que hacen los profesionales de los medios de comunicación de España se convierten en objeto de estudio esencial para comprender el impacto real que las redes sociales están teniendo en esta profesión.

Frente a la caracterización inicial por parte de los propios periodistas de las redes sociales como una moda pasajera, nos encontramos en una fase de adopción de estas herramientas convertidas en canales alternativos que, para los periodistas, son herramientas básicas y complementarias en sus trabajos diarios. Sin embargo, el nivel de fiabilidad que les otorgan es significativamente menor que la confianza que tienen en los medios de comunicación tradicionales. A pesar de estas incertidumbres, los medios periodísticos están poniendo en marcha diferentes acciones para posicionarse dentro de estas plataformas. Poco a poco, los medios de comunicación españoles han ido fomentando entre sus periodistas una cultura 2.0 basada en la integración de las redes sociales a su trabajo.

A pesar de que aún queda mucho por investigar acerca de estos nuevos canales, su eclosión en el periodismo supone un punto de inflexión en la forma de difundir noticas, buscar informaciones o interaccionar con el público. Por ello, y aunque es muy pronto para medir las consecuencias reales de las diferentes acciones que se llevan a cabo en el seno de los medios de comunicación con respecto a estas plataformas, lo que es evidente es que los periodistas están desempeñando un rol cada vez más visible dentro de estos canales sociales.

El último informe The State of the News Media 2013 señala que cada vez son más los usuarios que se informan a través de internet y de las redes sociales, haciendo uso en gran medida de los dispositivos móviles (Pew Research Center, 2013). Esta situación es aprovechada por organizaciones privadas, instituciones políticas y grupos de presión que utilizan de forma constante las herramientas que ofrece la Web 2.0 para hacer llegar sus mensajes a los ciudadanos, sin que haga falta que los medios de comunicación sean sus intermediarios o filtren ese mensaje. Como señalaba Diezhandino (2007, p.150)

El periodismo se ha abandonado a la corriente más fácil. Se ha rendido al creador de noticias (newsmaker). Ya no parece necesitarse ese viejo 'olfato periodístico' propio del buscador de noticias. Se las ofrecen en bandeja de plata. Por eso, la característica actual es la reproducción, por los distintos canales noticiosos, de los mismos temas.

Internet ha hecho que el acceso a fuentes directas por parte de los ciudadanos sea más habitual, si bien ahora existe una descompensación entre las medidas y estrategias adoptadas por los medios para llegar a la opinión pública y las de otras organizaciones no periodísticas. Mientras que las grandes compañías invierten en departamentos de comunicación formados por periodistas, especialistas en medios sociales y participación, los medios están reduciendo drásticamente las plantillas y los presupuestos, una situación que termina repercutiendo en la calidad periodística de los contenidos informativos, que en numerosas ocasiones es publicity que se presenta como información y que llega desde fuentes interesadas.

Esta situación parece haber aumentado con la aparición de las redes sociales, dado que cada vez son más numerosos los canales de los que disponen los usuarios y las organizaciones para emitir sus mensajes. La labor del periodista a la hora de filtrar, jerarquizar y simplificar esa ingente cantidad de datos que circula por la red es más importante que nunca. En palabras de Lippman (1922, p.38) "no puede haber libertad en una comunidad que carece de la información necesaria para detectar la mentira". Por tanto, el futuro de la profesión dependerá, en gran medida, de la fortaleza que muestren las instituciones periodísticas y su rapidez de respuesta a la hora de distinguir cuál es la información valiosa para el público, aquella que precisamente permite detectar mentiras. 
Internet y las redes sociales han hecho, en cierta manera, más transparentes las deficiencias de la profesión (limitada agenda propia, homogeneización de contenidos, ruedas de prensa sin preguntas, comparecencias políticas a través de pantallas de televisión, noticias que luego resultan ser falsas...), pero las oportunidades también son muchas y el panorama informativo se encuentra en plena ebullición. El papel del periodismo es fundamental en nuestra sociedad.

\section{Las redes sociales transforman las dinámicas periodísticas}

El mundo académico, a nivel nacional e internacional, muestra un fuerte consenso a la hora de afirmar que las nuevas plataformas comunicativas modifican el entorno laboral y las propias funciones de los periodistas. Los nuevos perfiles emergentes o la necesidad de un periodista polivalente dentro de las redacciones han sido los temas más analizados en los últimos años en relación a la profesión periodística y los nuevos medios (Bradshaw, 2007; Briggs, 2007; Herreros \& Vivar, 2007; Scolari, 2008; Bennett, 2011; García, 2012; Diezhandino, 2012). Todos estos trabajos reflexionan sobre las nuevas características de la comunicación digital: multimedialidad, hipertextualidad, gestión de los espacios colaborativos, nuevas formas de participación..., que para estos autores (en consonancia con la opinión de profesionales del sector) exigen un perfil del periodista más flexible para adaptarse a un entorno en constante cambio.

Aunque la profesión periodística no gire en torno a las redes sociales, cualquier periodista como profesional de la comunicación y por tanto con una serie de intereses públicos debe estar al corriente de la conversación ${ }^{3}$ que está teniendo lugar en el seno de estos espacios públicos. Por su parte, Skoler $(2009$, p.39) considera que en "las redes sociales no se hace periodismo" sino que a veces hay temas o noticias que surgen en estos lugares que pueden ser de interés periodístico. En ambos casos, el periodista debe atender a lo que ocurre en estos espacios sociales y utilizarlos de forma diligente, ya que estos canales han mostrado potencial informativo para poder hacer buen periodismo a través de ellos: Hay que agregar que no todos los usuarios que participan en estas redes lo hacen desde el punto de vista informativo.

Las redes sociales han ampliado la cobertura de ciertos acontecimientos que, de otro modo, se hubieran limitado a los canales tradicionales.

El actual panorama informativo y comunicativo al que nos enfrentamos es parte de una fase caracterizada por la interactividad, la participación de las audiencias o la desintermediación, los cuales acompañan a la pérdida del monopolio de la emisión pública por parte de los medios de masas tradicionales, si bien estos siguen siendo actores clave en el proceso comunicativo. Conceptos de los que ahora se habla como feedback o interactividad no son nuevos en la teoría de la comunicación sino que han formado parte del debate sobre los medios de masas y el periodismo prácticamente desde su aparición. Lo que cambia es la forma en la que estos elementos/conceptos teóricos se organizan o interaccionan entre sí dentro de un entorno digital que se encuentra en continua evolución.

\section{Procedimientos metodologicos}

Nuestro universo estáconstituido por los periodistas españoles; sin embargo, por tratarse de una población de gran tamaño, hemos acotado nuestro universo a los periodistas titulados. En España existen 75346 periodistas desde que en 1976 se graduaran los primeros licenciados en Ciencias de la Información. Así, nuestra muestra ha quedado limitada a 410 periodistas con un margen de error de $+/-5 \%$ y un coeficiente de confianza del $95 \%$ tratándose por tanto de una muestra no probabilística e intencional, ya que incluye ciertas características específicas y deja afuera a aquellos que no cumplen esos criterios.

Una vez decidido el tamaño muestral, y para que este fuera representativo, seleccionamos los sujetos que integrarían la muestra. La técnica que se llevó a cabo fue un muestreo estratégico o de conveniencia, no probabilístico, en el que la selección de unidades muestrales responde a los criterios acordes con la investigación.

\footnotetext{
${ }^{3}$ La conversación que se genera en las redes sociales contribuye, como ya señalaba Gabriel Tarde en 1904, a reforzar la opinión pública sobre determinados acontecimientos.
} 
Hemos evaluado diferentes factores, como la naturaleza del fenómeno a estudiar, nuestros objetivos de investigación y nuestros recursos, tanto humanos como económicos, con los que contábamos para llevarla a cabo.

Por tratarse del instrumento más idóneo para abordar uno de los principales objetivos de la investigación - conocer cuál es el uso que los periodistas españoles están haciendo de las redes sociales en su trabajo informativo -, la técnica cuantitativa utilizada en el trabajo de campo ha sido la encuesta. Al tratarse de una encuesta online, y aunque la rigurosidad de este método debe ser la misma que para un cuestionario tradicional, se han tomado como referencia los estándares de calidad para realizar encuestas por internet propuestos por la Asociación Alemana de Institutos de Mercado y Opinión (ADM) ${ }^{4}$. Antes de administrar el cuestionario final, la batería de preguntas ha sido sometida a las diferentes pruebas de validez y fiabilidad que permiten que nuestra técnica pueda ser replicada en el futuro por cualquier otro investigador. Para alcanzar niveles óptimos de validez de contenido se ha utilizado la técnica de jueces expertos. Para ello, se envió el cuestionario a un grupo de 14 jueces expertos. Una vez que aumentamos la validez con el pretest del cuestionario, pasamos a comprobar su fiabilidad a través del test-retest. En general se observaron índices de fiabilidad muy altos, Kappa =0,70 en el peor de los casos.

La encuesta online que diseñamos bajo el título de "Periodismo y Redes Sociales"fue alojada en el servidor de encuestas online e-encuesta ${ }^{5}$ el día 27 de julio de 2011 y se mantuvo abierta hasta el 8 de mayo de 2012. La información sobre el enlace que daba acceso a la encuesta se hizo a través de un texto de presentación, en el que se pedía a periodistas licenciados que participaran en la misma. El cuestionario definitivo consta de 14 preguntas que agrupan 58 variables. La encuesta combina preguntas de opción múltiple, cerradas y de escala likert.

El número total de cuestionarios que se cumplimentaron fue de 517, de los cuales se descartaron aquellos que no habían contestado, como mínimo, al $70 \%$ del cuestionario (8 preguntas de 14); de esta manera, se han considerado inválidas 107 encuestas. El análisis de datos se ha hecho sobre 410 periodistas. A continuación pasamos a la exposición detallada de los resultados.

\section{Resultados y Discusión}

Facebooky Twitter son las redes sociales en las que un mayor número de periodistas tiene una cuenta abierta. Un 90,73\% de los periodistas encuestados está en Facebook y un 88,29\% en Twitter (Tabla 1). El hecho de que sean estas redes sociales donde más periodistas hay registrados no significa que sean las más utilizadas dentro de su profesión. De hecho, la gente tiende a abrirse un perfil en más de dos redes sociales, pero su mayor actividad se suele concentrar en una de ellas. Estas dos redes sociales cuentan con una mayor presencia de los profesionales de la información. Aunque Twitter es la plataforma más popular entre los periodistas (Asociación Española de la Economía Digital, 2012; Gámez, 2012; Oriella PR Network, 2011), Facebook se encuentra por delante, de forma poco significativa, ya que a nivel mundial concentra el mayor número de usuarios.

Según el último informe sobre la Sociedad de la Información, el 95,00\% de los usuarios de redes sociales tienen un perfil abierto en Facebooky tres de cada cuatro

Tabla 1. Redes sociales usadas por periodistas.

\begin{tabular}{lcc}
\hline Red social & Frecuencia & $\%$ \\
\hline Facebook & 372 & 90,73 \\
Twitter & 362 & 88,29 \\
Linkedln & 247 & 60,24 \\
Google+ & 120 & 29,27 \\
Tuenti & 103 & 25,12 \\
Otras & 46 & 11,22 \\
MySpace & 24 & 5,85 \\
Ninguna & 9 & 2,20 \\
\hline
\end{tabular}

Fuente: Elaboración propia (2012).

\footnotetext{
${ }^{4}$ El documento fue redactado en mayo de 2001 y se puede consultar una versión traducida al español dedicho documento em: <http://www.netquest.com/ papers/Onlinestandards_ES.pdf>.

${ }^{5}$ El servidor ofrece una versión básica y gratuita de hasta cien encuestas. Nosotros trabajamos con la versión profesional y de pago que nos permitía recopilar hasta 500 encuestas más; es decir, con las 100 iniciales teníamos un límite de 600 encuestas, lo que se ajustaba a nuestras necesidades.
} 
internautas españoles acceden a ella diariamente (Fundación Orange, 2012). En cuanto al resto de las redes, las más utilizadas por los internautas españoles según el mismo informe son Twittery Tuenti. La red Tuenti no es la que más destaca en el entorno periodístico $(25,12 \%)$, entre otras cosas porque se trata de una red juvenil enfocada principalmente a los adolescentes y la estrategia de los medios en esta red pasa por cubrir estas necesidades juveniles con contenidos ajustados a sus gustos e intereses. De hecho, los usuarios de esta red social disminuyen a medida que se incrementa la edad del internauta.

Otras plataformas sociales como Google + tienen un porcentaje del 29,70\%. La red MySpace es en la que menos perfiles abiertos tienen los periodistas encuestados (5,85\%). Es posible que su especialización en música la convierta en una plataforma interesante para un grupo específico de profesionales, como pueden ser los periodistas especializados en música, pero no ha resultado tan atractiva para otros profesionales de corte más generalista.

Después de haber constatado que la mayoría de los periodistas encuestados pertenece a una red social como mínimo y que algunas de ellas, como Twitter, aparecen muy bien posicionadas a la hora de buscar información, quisimos saber si las redes sociales se están convirtiendo, hoy en día, en una herramienta más en el entorno periodístico. Para ello, uno de nuestros objetivos era conocer en qué medida los profesionales encuestados utilizan las redes sociales en su trabajo como periodistas.

El (225) de los encuestados asegura utilizarlas siempre (esto es muy frecuentemente) y un (137), a veces (Figura 1). Estos porcentajes nos están indicando que casi un $90 \%$ de los periodistas encuestados utiliza las redes sociales en su trabajo; porcentajes muy significativos si tenemos en cuenta que solo un (14) de los periodistas encuestados dice no utilizarlas en su trabajo periodístico y un (30) señala usarlas poco (entendiendo que dentro de la escala Likert poco es "rara vez").

Una vez que hemos confirmado que los periodistas utilizan las redes sociales en su trabajo, la siguiente cuestión que nos planteamos es para qué las usan (Figura 2). Los principales usos que los periodistas españoles dicen hacer de las redes sociales en su trabajo son: difundir información de su propio medio, conocer la opinión de los usuarios, detectar nuevos temas, buscar información y contactar con fuentes. Otros usos que los periodistas han señalado son atraer audiencia y contrastar informaciones. Algunos de estos usos coinciden con los encontrados en estudios anteriores sobre el mismo objeto (Carrera et al., 2012).

Algo de destacar en estos resultados es que los principales usos que hacen los periodistas de las redes sociales responden, en primer lugar, a una estrategia de promoción de su propio trabajo y, en segundo a una labor propiamente informativa. Hablan de difundir información del medio para el que trabajan, publicitando sus propios contenidos o los de otros compañeros del mismo medio y de conocer la opinión de la gente (sondeos). Las tareas

\section{Uso de las redes sociales}

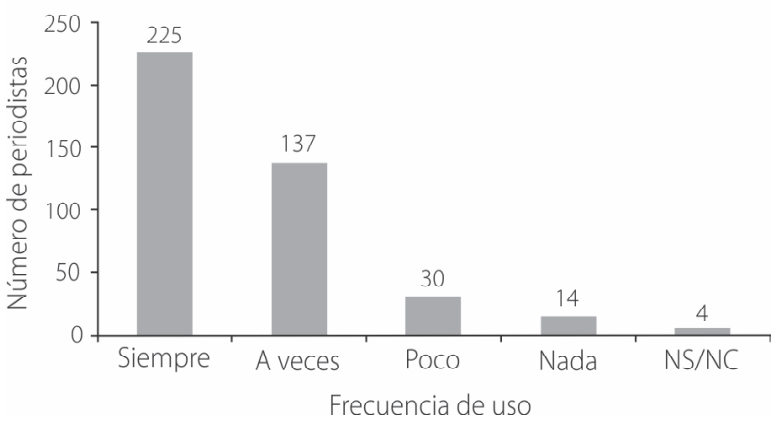

Figura 1. Frecuencia de uso de las redes sociales.

Fuente: Elaboración propia (2012).

Nota: $\mathrm{NC}=$ No contesta; $\mathrm{NS}=$ No sabe

Para qué utiliza usted las redes sociales en su trabajo

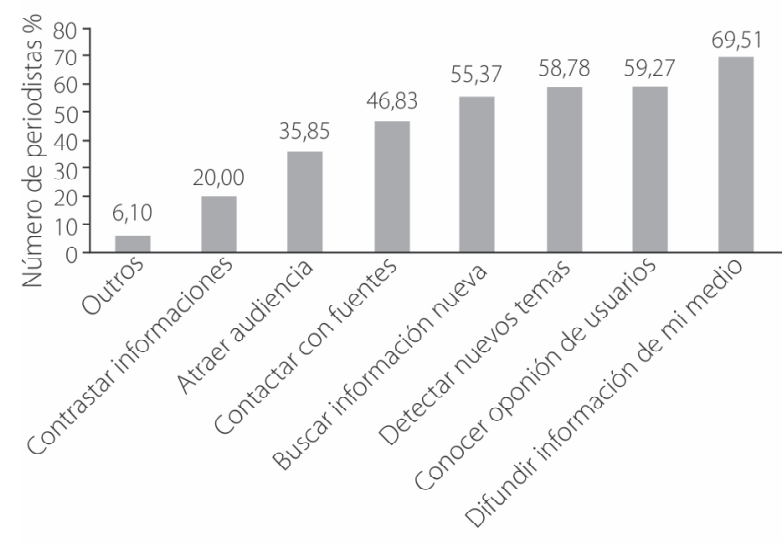

Usos que les periodistas hacen de las redes sociales

Figura 2. Principales usos de las redes sociales.

Fuente: Elaboración propia (2012). 
más periodísticas que han señalado son las de buscar nuevos temas e informaciones y contactar con fuentes. El uso que los periodistas españoles hacen en menor medida de estas plataformas es el de contrastar informaciones.

Cuando se pregunta a los periodistas si las redes sociales son más o menos fiables que los medios de comunicación tradicionales, o si gozan de la misma credibilidad que ellos, los resultados son significativos: un (274) de los periodistas encuestados afirman que las

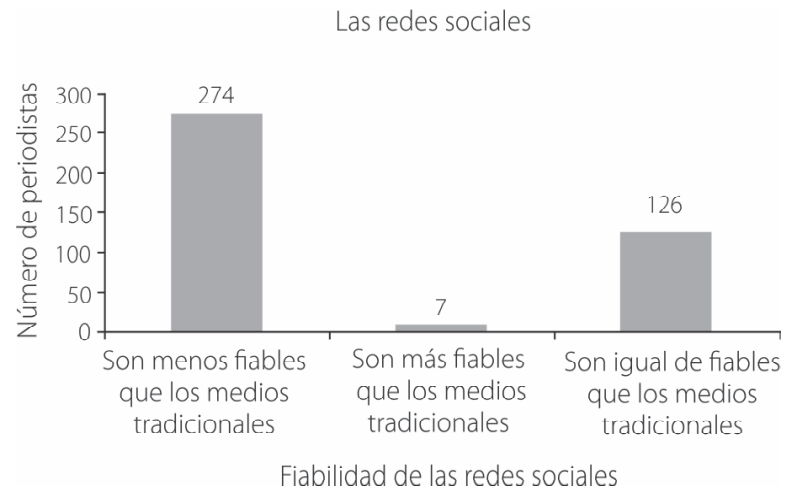

Figura 3. Fiabilidad de las redes sociales.

Fuente: Elaboración propia (2012).

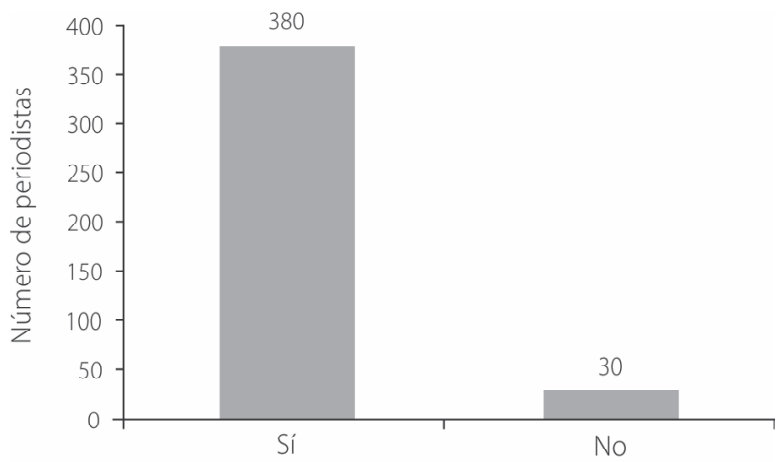

Respuesta de los periodistas

Figura 4. ¿Son las redes sociales una herramienta periodística más? Fuente: Elaboración propia (2012).

\section{Referencias}

Asociación Española de la Economía Digital. Uso de twitter en España 2012. Madrid: Adigital, 2012.

Bennett, L.W. News: The politics of illusion. Washington: Pearson, 2011.

Bradshaw, P. Un modelo para la redacción del siglo XXI. Cuadernos de Periodistas, v.12, p.74-79, 2007. redes sociales son menos fiables que los medios tradicionales, un (126) dice que son igual de fiables y solo un (7) cree que las redes sociales son más fiables que los medios tradicionales (Figura 3).

A pesar de esta opinión, gran parte de los periodistas encuestados considera que las redes sociales son una herramienta periodística valiosa (Figura 4). Un (38) señala que los social media son una herramienta dentro del periodismo, frente a un (30) que señala que no son un instrumento periodístico. Cabe indicar que este $7 \%$ está conformado por periodistas de todas las edades y, por tanto, la respuesta no está condicionada por la edad de los periodistas.

\section{Conclusión}

La mayoría de los periodistas españoles pertenecen a más de una red social. Facebook y Twitter se posicionan como las más usadas entre los periodistas encuestados. Facebook es utilizada por un $90,73 \%$ y la red de microblogging por un $88,29 \%$ de los profesionales.

Los principales usos que los periodistas españoles hacen de las redes sociales son: difundir información del medio en el que trabajan, conocer la opinión de los usuarios, detectar nuevos temas, buscar información y contactar con fuentes.

Promocionar sus propios trabajos, o el de los compañeros, es otro de los usos más característicos que hacen los periodistas de las redes sociales. La función viral que tienen los Social Media parece ser una de las potencialidades que más atracción despierta entre los periodistas.

La credibilidad que los profesionales de la información encuestados atribuyen a las redes sociales continúa siendo baja, a pesar de que dichas plataformas llevan tiempo instauradas en las rutinas de los periodistas y estos las consideran una herramienta más en su trabajo diario.

Briggs, M. Journalism 2.0. Austin: Knight Center, 2007

Carrera, P., et al. Journalism \& social media: How spanish journalists are using twitter. Estudios sobre el Mensaje Periodístico, v.18, n.1, p.31-53, 2012.

Diezhandino, M.P. Periodismo y poder. Madrid: Pearson, 2007. 
Diezhandino, M.P. El periodista en la encrucijada. Barcelona: Fundación Telefónica, 2012.

Fishman, M. La fabricación de la noticia. Buenos Aires: Tres Tiempos, 1983.

Fundación Orange. eEspaña: informe anual 2012 sobre el desarrollo de la sociedad de la información en España. Madrid: Fundación Orange, 2012. Disponible en: <http:// fundacionorange.es/fundacionorange/analisis/eespana/ e_espana12.html >. Acceso en: 5 de enero de 2013.

Gámez, D. El periodismo en Twitter. In: Gámez, D. Twitter: cómo 140 caracteres pueden ayudarle a mejorar su presencia online. Barcelona: Profit Editorial, 2012. p.85-120.

Gans, H.J. Deciding what's news: A study of CBS evening news, NBC nightly news, Newsweek and Time. New York: Pantheon, 1979.

García, N. Redes sociales y periodismo: de la información a la comunicación. In: García, N. Redes sociales en Internet: implicaciones y consecuencias de las plataformas 2.0 en la sociedad. Madrid: Universitas, 2012. p.213-239.

Gieber, W. Across the desk: A study of 16 telegraph editors. Journalism Quarterly, v.33, n.4, p.423-433, 1956.

Herreros, M.C.; Vivar, J.F. Blogs y periodismo en la red. Madrid: Fragua, 2007.

Lewin, K. Channel of group life. Human Relations, v.1, n.2, p.143-153, 1947.
Lippman, W. Public opinion. New York: Harcourt, 1922.

McQuail, D. Introducción a la teoría de la comunicación de masas. Barcelona: Paidoìs, 2000.

Oriella PR Network. The State of journalism in 2011. Oriella PR Network Digital Journalism Study, 2011. Available from: <http:// www.orielladigitaljournalism.com/files/assets/downloads/ publication. pdf>. Cited: Jan. 8, 2013.

Pew Research Center'S Project. The state of the news media 2013. Washington: The Pew Research Center's, 2013. Available from: <http://www.stateofthemedia.org/2013/overview-5/>. Cited: Jan. 8, 2013.

Robinson, G. News agencies and world news: In Canada, the United States, and Yugoslavia: Methods and data. Fribourg: University of Fribourg, 1981.

Scolari, C. Hipermediaciones: elementos para una teorìa de la comunicacioìn digital interactiva. Barcelona: Gedisa, 2008.

Skoler, M. Why the news media became irrelevant and how social media can help. Cambridge: Nieman Reports, 2009.

Tuchman, G. Making news. New York: Free Press, 1980.

White, D. The Gatekeeper: A case-study in the selection of news. Journalism Quarterly, v.27, n.3, p.383-390, 1950.

Wolf, M. La investigacioin de la comunicacioin de masas. Barcelona: Paidoìs, 1987. 

